\title{
A programmable DNA origami platform to organize SNAREs for membrane fusion
}

Weiming $\mathrm{Xu}^{1,2}$, Bhavik Nathwani ${ }^{3}$, Chenxiang Lin ${ }^{3 \#}$, Jing Wang ${ }^{1,2}$, Erdem Karatekin ${ }^{2,4,5,6}$, Frederic Pincet $^{1,2,7}$, William Shih ${ }^{3^{*}}$, and James E. Rothman ${ }^{1,2^{*}}$

\section{Materials and Methods}

$\underline{\text { General reagents and abbreviations }}$

n-Octyl-beta-D-Glucopyranoside (OG)

Dithiothreitol (DTT)

2,2'-Dithiodipyridine (DTDP), Sigma-Aldrich, 43791

Tris(2-carboxyethyl) phosphine hydrochloride (TCEP, Sigma-Aldrich, C4706)

OptiPrep, composed of $60 \%$ (w/v) solution of iodixanol in water (sterile) (Sigma-Aldrich, D1556,)

All the synthetic phospholipids were purchased from Avanti Polar Lipids, and were used without further purification, including:

1,2-dioleoyl-sn-glycero-3-phosphocholine (DOPC, Cat. \# 850375C)

1,2-dioleoyl-sn-glycero-3-phospho-L-serine (sodium salt) (DOPS, Cat. \# 840035C)

1,2-dioleoyl-sn-glycero-3-phosphoethanolamine-N-[methoxy(polyethylene glycol)-2000] (ammonium salt) (PEG2000PE, Cat. \# 880130C)

1,2-dioleoyl-sn-glycero-3-phosphoethanolamine-N-(7-nitro-2-1,3-benzoxadiazol-4-yl) (ammonium salt) (NBD-DOPE, Cat. \# 810145C)

1,2-dioleoyl-sn-glycero-3-phosphoethanolamine-N-(lissamine rhodamine B sulfonyl) (ammonium salt) (Rhod-DOPE, Cat.\# 810150C)

1,2-dioleoyl-sn-glycero-3-phosphoethanolamine-N-[4-(p-maleimidophenyl)butyramide] (sodium salt) (Maleimide-PE, Cat. \# 870012C) 


\section{Protein purification}

Cys free t-SNARE complexes of rat Syntaxin 1A and mouse SNAP25 were derived from plasmid pTW34, and were expressed and purified as previously described. ${ }^{1}$ Cys free mouse VAMP2 (pet-SUMO-VAMP2, C103S) and Cys-VAMP2 (pet-SUMO-VAMP2, S2C, C103S) was derived from wild type VAMP2 (pet-SUMO-VAMP2) and were expressed and purified as previously described. ${ }^{2}$

\section{Origami ring preparation ( to prepare (e) in Figure 1)}

The DNA ring was designed by caDNAno software. For the DNA ring to self-assemble, a solution containing p8064 scaffold strand $(50 \mathrm{nM})$, staple strands ( $300 \mathrm{nM}$ each, synthesized by Integrated DNA Technologies, Inc.), and $1 \times$ folding buffer $(5 \mathrm{mM}$ Tris, $1 \mathrm{mM}$ EDTA, $12 \mathrm{mM}$ $\mathrm{MgCl}_{2}, \mathrm{pH}$ ) ) was subject to a thermal-annealing ramp $\left(80{ }^{\circ} \mathrm{C}-65^{\circ} \mathrm{C}-1{ }^{\circ} \mathrm{C} / 5 \mathrm{~min} ; 64{ }^{\circ} \mathrm{C}-24{ }^{\circ} \mathrm{C}-1\right.$ ${ }^{\circ} \mathrm{C} / 50 \mathrm{~min} ; 15^{\circ} \mathrm{C}$ hold). Well-folded DNA rings were purified by a rate-zonal centrifugation procedure using a $15-45 \%(\mathrm{v} / \mathrm{v})$ glycerol gradient. The purified DNA rings were concentrated to $50 \mathrm{nM}$ (concentration measured by UV absorbance at $260 \mathrm{~nm}$ ) and stored at $-20^{\circ} \mathrm{C}$ in $1 \times$ folding buffer.

3. Cross-linking of "Anti handle i" oligonucleotide to Cys-VAMP2 protein (to prepare (c) in Figure 1)

HPLC purified Thiol-5' -anti handle i-3' sequences, with an Alexa647 label on the 3' end, were purchased from IDT and used without further purification.

$50 \mu \mathrm{L}$ of protein, Cys-VAMP2, solution (about $90 \mu \mathrm{M} / \mathrm{mL}$ in $25 \mathrm{mM}$ HEPES, pH 7.4, $400 \mathrm{mM}$ $\mathrm{KCl}, 0.2 \mathrm{mM}$ TCEP, 10\% Glycerol, 1\% OG) was reduced using $2 \mathrm{mM}$ DTT (final concentration) for about $1 \mathrm{hr}$ at RT, and then buffer exchanged into buffer A (100 mM NaH $2 \mathrm{PO}_{4} / \mathrm{Na}_{2} \mathrm{HPO}_{4}, \mathrm{pH}$ 5.8, $400 \mathrm{mM} \mathrm{NaCl}, 1 \% \mathrm{OG}$ ) using Micro Bio-Spin 6 chromatography columns (Bio-Rad). The resulting protein solution was immediately mixed with $1 / 10$ volume of concentrated DTDP (Sigma-Aldrich, 43791) stock solution (10 mM DTDP in buffer A with 25\% acetonitrile) so that the final concentration of DTDP in solution was about $1 \mathrm{mM}$. The reaction was allowed to proceed for 30 mins. at RT. Excessive amount of DTDP was then removed by two passages through Micro Bio-Spin columns pre-equilibrated with buffer B $\left(100 \mathrm{mM} \mathrm{NaH} \mathrm{PO}_{4} / \mathrm{Na}_{2} \mathrm{HPO}_{4}\right.$, pH 8.0, $400 \mathrm{mM} \mathrm{NaCl}, 1 \%$ OG).

$50 \mu \mathrm{L}$ of thiol-5'-anti handle i-3' solution $\left(500 \mu \mathrm{M} / \mathrm{mL}\right.$ in $\left.\mathrm{H}_{2} \mathrm{O}\right)$ was reduced with $10 \mathrm{mM}$ DTT (final concentration) for about $2 \mathrm{hrs}$ at RT (this step can be done at the same time as the DTT 
treatment of protein), and then buffer exchanged into $\mathrm{B}\left(100 \mathrm{mM} \mathrm{NaH}_{2} \mathrm{PO}_{4} / \mathrm{Na}_{2} \mathrm{HPO}_{4}, \mathrm{pH} 8.0\right.$, $400 \mathrm{mM} \mathrm{NaCl}, 1 \%$ OG) using Micro Bio-Spin 6 chromatography columns (Bio-Rad). The resulting oligonucleotide molecules were immediately mixed with above thiol-pyridine activated protein solution. The reaction was allowed to proceed for about $20 \mathrm{hrs}$ at RT.

After the cross-linking reaction, excessive free anti handle sequences were isolated from the reaction mixture by gel filtration, using a Superdex 75 10/300 GL column (GE Healthcare) on a AKTA purifier (GE Healthcare). The column was eluted with $25 \mathrm{mM}$ HEPES, pH 7.4, $400 \mathrm{mM}$ $\mathrm{KCl}, 1 \% \mathrm{OG}$, at a flow rate of $0.5 \mathrm{~mL} / \mathrm{min}$. Fractions containing product, identified by SDS gels, were combined. Concentration of the final product was determined by absorbance at $647 \mathrm{~nm}$. Product solution was then snap frozen in presence of $10 \%$ glycerol, and stored in $-80{ }^{\circ} \mathrm{C}$ for further use. The final product was named as "Alexa647-anti handle i-VAMP2".

4. Cross-linking of "Anti handle ii" oligonucleotide sequence to Maleimide-DOPE (to prepare (d) in Figure 1)

HPLC purified Thiol-5' -anti handle ii-3' sequences, with an Alexa488 label on the 3' end, were purchased from IDT and used without further purification.

In test tube A, total of $1 \mu$ mole phospholipid (in chloroform as a clear solution) containing (mole\%) 79.2\% of DOPC, $15 \%$ of DOPS, $5 \%$ of PEG 2000 -PE and $0.8 \%$ of Rho-DOPE, was dried under Nitrogen flow and then in vacuum for at least 2 hours. In test tube B, total of 20 nmoles of Maleimide-DOPE (in chloroform as a clear solution) was dried under Nitrogen flow and then in vacuum for at least 1 hours.

$22.5 \mu \mathrm{L}$ of thiol-5' -anti hadle ii-3' solution $\left(500 \mu \mathrm{M} / \mathrm{mL}\right.$ in $\left.\mathrm{H}_{2} \mathrm{O}\right)$ was first de-protected using 2.5 $\mu \mathrm{L}$ of $200 \mathrm{mM}$ Tris(2-carboxyethyl) phosphine hydrochloride (TCEP, Sigma-Aldrich, C4706) for about 1 hour at RT, and then buffer exchanged to $25 \mathrm{mM}$ HEPES.KOH, $\mathrm{pH} 7.4,400 \mathrm{mM}$ $\mathrm{KCl}, 0.2 \mathrm{mM}$ TCEP, using Micro Bio-Spin 6 chromatography columns.

In test tube B, the dried lipid film was resuspended with total of $200 \mu \mathrm{L}$ of solution containing above pre-treated oligonucleotide, final of $2 \%$ OG and buffer, $25 \mathrm{mM}$ HEPES.KOH, pH 7.4, 400 $\mathrm{mM} \mathrm{KCl}, 0.2 \mathrm{mM}$ TCEP. The mixture was vortexed for $30 \mathrm{~min}$ at RT and then another $30 \mathrm{mins}$ standing on bench (no shaking) at RT to allow the coupling reaction proceed. $10 \mu 1$ of $140 \mathrm{mM}$ $\beta$-mercapitoethanol was then added into the system and incubated at RT for another 10 mins to quench further reaction. All the mixture was then transferred into test tube A. Test tube A was then vortexed for another 20 mins at RT to allow complete resuspension of dried lipid film into solution. 
Into test tube A, $800 \mu \mathrm{L}$ of buffer, $25 \mathrm{mM}$ HEPES.KOH, $\mathrm{pH} 7.4,400 \mathrm{mM} \mathrm{KCl}$, was added while being vortexed, so that the final detergent OG concentration went down to $0.4 \%$, below its CMC. OG was then removed by dialyzing the mixture against $2 \mathrm{~L}$ of buffer, $25 \mathrm{mM}$ HEPES.KOH, $\mathrm{pH}$ 7.4, $400 \mathrm{mM} \mathrm{KCl}$, overnight at RT, in presence of $2 \mathrm{~g}$ of SM-2 beads (Bio-rad, 152-3920).

Cross-linked product (now reconstituted in liposomes) was then separated from un-reacted oligonucleotide by the technique, isopycnic centrifugation. During the centrifugation, liposome containing the product floated up and equilibrated at the low-density layer, while the oligonucleotide equilibrated at the high-density layer at the bottom of the tube. To this purpose, a three-layer step-gradient was prepared in a Beckman centrifuge tube (Beckman, 349622) using OptiPrep as the medium (Sigma-Aldrich, D1556, composed of $60 \%(\mathrm{w} / \mathrm{v})$ solution of iodixanol in water (sterile)). Top layer, $750 \mu \mathrm{L}$ of buffer, $25 \mathrm{mM}$ HEPES.KOH, $\mathrm{pH}$ 7.4, $400 \mathrm{mM} \mathrm{KCl}$, was first loaded into the centrifuge tube. Then, the middle layer, $750 \mu \mathrm{L}$ of a uniform mixture of $20 \%$ iodixanol (vol/vol, 1:2, OptiPrep:buffer), was loaded from the bottom (push needle against the bottom of the tube). Lastly, the bottom layer containing a uniform mixture of $1 \mathrm{~mL}$ of OptiPrep and $1 \mathrm{~mL}$ of the dialysis recovery solution was loaded from bottom. This tube of gradient mixture was then centrifuged at $50 \mathrm{~K}$ for $5 \mathrm{hrs}$ at $4^{\circ} \mathrm{C}$ on a SW55 rotor. After centrifugation, final product stayed at the interface between the top and the middle layer. Two portions of $150 \mu \mathrm{L}$ solution was collected (total of about $300 \mu \mathrm{L}$ ) at the interface and combined. Concentration of the final product was determined by absorbance at $488 \mathrm{~nm}$. Product solution was then snap frozen, and stored in $-80{ }^{\circ} \mathrm{C}$ for further use. The final product was named as "Alexa488-anti handle ii-DOPE".

5. Origami ring-VAMP2, DOPE complex preparation and separation (to prepare (f) in Figure 1)

Origami ring-VAMP2, DOPE complex was formed by mixing individual component, namely origami ring, Alexa647-anti handle i-VAMP2, and Alexa488-anti handle ii-DOPE, followed by incubation at $37{ }^{\circ} \mathrm{C}$ for $2 \mathrm{hrs}$. The reaction was carried out using 0.3 pmole origami ring. For each 1 eq. of origami ring bearing $n$ inner handles, excessive, $3 n$ eq., of Alexa647-anti handle iVAMP2, and $3 n$ eq. of Alexa488-anti handle ii-DOPE were used to push the hybridization reaction to completion. Final volume of the reaction mixture was adjusted to $50 \mu \mathrm{L}$ and in final buffer, $25 \mathrm{mM}$ HEPES.KOH, pH 7.4, $400 \mathrm{mM} \mathrm{KCl,} 10 \mathrm{mM} \mathrm{MgCl}$, 1\% OG and 10\% Glycerol.

The origami ring-VAMP2, DOPE complex was then separated from unreacted Alexa647-anti handle i-VAMP2, unreacted Alexa488-anti handle ii-DOPE, and lipids (came from the liposome that were used to purify Alexa488-anti handle ii-DOPE) by rate-zonal centrifugation, using 
glycerol as the medium. In a centrifuge tube (Beckman, \# 344090), step gradient (all layers are in buffer, $25 \mathrm{mM}$ HEPES.KOH, pH 7.4, $400 \mathrm{mM} \mathrm{KCl,} 10 \mathrm{mM} \mathrm{MgCl} 2,1 \%$ OG) was prepared by first laying $50 \mu \mathrm{L}$ of $60 \%$ glycerol layer as a cushion on the bottom of the centrifuge tube, and then on top of this cushion layer, laying 10 layers, with $50 \mu \mathrm{L}$ of each, containing from $40 \%$ glycerol layer on the bottom to $13 \%$ glycerol layer on the top with 3\% increment. This 11-layer glycerol step gradient medium was put in cold room overnight before use. Lastly, above $50 \mu \mathrm{L}$ of reaction mixture (in the same buffer as the gradient medium) containing $10 \%$ glycerol was laid on top of the gradient. This tube of gradient mixture was then centrifuged at $50 \mathrm{~K}$ for $1 \mathrm{hr} 20$ mins at $4{ }^{\circ} \mathrm{C}$ on a $\mathrm{SW} 55$ rotor.

After centrifugation, 12 fractions, with $50 \mu \mathrm{L}$ of each, were carefully collected from the top to the bottom. Fractions corresponding to the product were determined by nucleic acid electrophoresis using a gel containing $1.5 \%$ agarose and $0.5 \%$ SDS. Since the final complex has both Alexa 488 and Alexa 647 on it, product bands can be visualized by scanning the gel on a Typhoon Scanner (GE). In all of our preparation, the final complex moved to fraction 7 and 8 , and other smaller particles remained in fraction 1 to 3 . Fractions containing the final product were then combined and stored at $4^{\circ} \mathrm{C}$ for further use.

6. Preparation of liposomes pre-equiped with DNA-lipid tethers (to prepare (g) in Figure 1) Liposomes containing ssDNA-lipid as tethers were prepared for the further reconstitution of Origami ring-VAMP2, DOPE complexes (SUV) and t-SNARE for the TIRF based assay.

All the HPLC purified oligo sequences were purchased from IDT and were used without further purification. The preparation procedure and reagents (buffers, columns etc.) are the same as described in section 4, except that different amount of starting materials (reactants) was used as listed in the following.

For "Tether i-liposome", that was later used to reconstitute t-SNARE (to form SBL): (a) in test tube $\mathrm{A}$, total of $1 \mu$ mole phospholipid (in chloroform as a clear solution) containing (mole\%) $79.2 \%$ of DOPC, $15 \%$ of DOPS, $5 \%$ of PEG 2000 -PE and $1 \%$ of NBD-DOPE, was dried (operations refer to section 4); (b) In test tube B, total of 5 nmoles of Maleimide-DOPE (in chloroform as a clear solution) was dried; (c) $35 \mu \mathrm{L}$ of thiol-5' -Tether i-3' stock solution (500 $\mu \mathrm{M} / \mathrm{mL}$ in $\mathrm{H}_{2} \mathrm{O}$ ) was treated with $3.5 \mu \mathrm{L}$ of $200 \mathrm{mM}$ TCEP.

For "Anti tether i-liposome", that was then used to form SUV bearing ring-VAMP2-PE complexes: (a) in test tube A, total of $1 \mu$ mole phospholipid (in chloroform as a clear solution) containing (mole $\%$ ) $79.2 \%$ of DOPC, $15 \%$ of DOPS, $5 \%$ of PEG 2000 -PE and $0.8 \%$ of Rho- 
DOPE, was dried (operations refer to section 4); (b) In test tube B, total of 1 nmoles of Maleimide-DOPE (in chloroform as a clear solution) was dried; (c) $7 \mu \mathrm{L}$ of 5'-Anti tether i-3'thiol stock solution $\left(500 \mu \mathrm{M} / \mathrm{mL}\right.$ in $\left.\mathrm{H}_{2} \mathrm{O}\right)$ was treated with $1.0 \mu \mathrm{L}$ of $200 \mathrm{mM}$ TCEP. $15 \mu \mathrm{L}$ of buffer was added into the solution before spinning to meet the minimum required volume of the Micro-spin column.

After separation of liposome containing DNA-lipd tethers from un-reacted oligonucleotides by isopycnic centrifugation (refer to section 4), two portion of $150 \mu \mathrm{L}$ (total of $300 \mu \mathrm{L}$ ) of product were collected.

\section{Reconstitution of t-SNARE to "tether i-liposome" (procedure in Figure S3A)} $200 \mu \mathrm{L}$ (out of $300 \mu \mathrm{L}$ from the float-up recovery) of "Tether i-liposome" was mixed with 0.3 nmoles of t-SNARE protein, OG stock solution, and buffer ( $25 \mathrm{mM}$ HEPES.KOH pH 7.4, 400 $\mathrm{mM} \mathrm{KCl}$ ), so that the final volume of this solution mixture is $500 \mu \mathrm{L}$ containing $0.66 \% \mathrm{OG}$ (detergent, right at its CMC) and the lipid to protein ratio is about 2000:1. This solution was then vortexed for $1 \mathrm{hr}$ at room temperature. Another $500 \mu \mathrm{L}$ of buffer, $25 \mathrm{mM}$ HEPES.KOH, pH 7.4, $400 \mathrm{mM} \mathrm{KCl}$, was then added into this solution while vortexing, and the final OG concentration reached $0.33 \%$ (below its $\mathrm{CMC}$ ). OG was then removed by dialyzing the mixture against $2 \mathrm{~L}$ of buffer, $25 \mathrm{mM}$ HEPES.KOH, pH 7.4, $400 \mathrm{mM} \mathrm{KCl}$, overnight at RT and in presence of $2 \mathrm{~g}$ of SM-2 beads (Bio-rad, 152-3920).

Final product, "t-Tether i-liposome" was then separated from any free-protein, as well as small amount of left-over un-reacted oligonucleotide by centrifugation. To this purpose, a three-layer step-gradient was prepared in a Beckman centrifuge tube (Beckman, 349622) using OptiPrep as the medium. Top layer, $750 \mu \mathrm{L}$ of buffer, $25 \mathrm{mM}$ HEPES.KOH, pH 7.4, $400 \mathrm{mM} \mathrm{KCl}$, was first loaded into the centrifuge tube. Then, the middle layer, $750 \mu \mathrm{L}$ of a uniform mixture of $20 \%$ iodixanol (in final $25 \mathrm{mM}$ HEPES.KOH, pH 7.4, $400 \mathrm{mM} \mathrm{KCl}$ ), was loaded from the bottom (push needle against the bottom of the tube). Lastly, the bottom layer containing a uniform mixture of $1 \mathrm{~mL}$ of OptiPrep and $1 \mathrm{~mL}$ of the dialysis recovery solution was loaded from bottom. This tube of gradient mixture was then centrifuged at $50 \mathrm{~K}$ for $5 \mathrm{hrs}$ at $4^{\circ} \mathrm{C}$ on a SW55 rotor. After centrifugation, final product stayed at the interface between the top and the middle layer. Two portions of $100 \mu \mathrm{L}$ solution was collected (total of about $200 \mu \mathrm{L}$ ) at the interface and combined for later TIRF experiment. 
8. Reconstitution of ring-VAMP2, DOPE complex to "Anti-tether i-liposome" (to prepare (h) in Figure 1)

$20 \mu \mathrm{L}$ (out of $300 \mu \mathrm{L}$ from the float-up recovery) of "Anti-tether i-liposome" was mixed with 40 $\mu \mathrm{L}$ of ring-VAMP2, DOPE complex obtained in (section 5). $0.2 \mu \mathrm{L}$ of $1 \mathrm{M} \mathrm{MgCl}_{2}$ stock solution was added into the mixture, so that the final buffer contains $25 \mathrm{mM}$ HEPES.KOH, pH 7.4, 400 $\mathrm{mM} \mathrm{KCl}, 10 \mathrm{mM} \mathrm{MgCl}_{2}$, and $0.66 \% \mathrm{OG}$. This solution was then vortexed for $1 \mathrm{hr}$ at room temperature. Another $60 \mu \mathrm{L}$ of buffer, $25 \mathrm{mM}$ HEPES.KOH, pH 7.4, $400 \mathrm{mM} \mathrm{KCl,} 10 \mathrm{mM}$ $\mathrm{MgCl}_{2}$, was then added into this solution followed by brief vortexing, and the final OG concentration reached $0.33 \%$ (below its $\mathrm{CMC}$ ). OG was then removed by dialyzing the mixture against $2 \mathrm{~L}$ of buffer, $25 \mathrm{mM}$ HEPES.KOH, pH 7.4, $400 \mathrm{mM} \mathrm{KCl}, 10 \mathrm{mM} \mathrm{MgCl}$, overnight at $\mathrm{RT}$ and in presence of $2 \mathrm{~g}$ of SM-2 beads (Bio-rad, 152-3920).

Final product, "ring complex-Anti tether i-liposome" was then separated from most excessive Anti tether i-liposome, as well as small amount of ring complex aggregates by centrifugation. To this purpose, a three-layer step-gradient was prepared in a Beckman centrifuge tube (Beckman, \# 344090) using OptiPrep as the medium (Sigma-Aldrich, D1556, composed of 60\% (w/v) solution of iodixanol in water (sterile)). The bottom layer containing a uniform mixture of 150 $\mu \mathrm{L}$ of dialysis recovery, $200 \mu \mathrm{L}$ of OptiPrep and $50 \mu \mathrm{L}$ of 5 xbuffer $(125 \mathrm{mM}$ HEPES.KOH, $\mathrm{pH}$ 7.4, $2 \mathrm{M} \mathrm{KCl}, 50 \mathrm{mM} \mathrm{MgCl}$ ) (OptiPrep and 5xbuffer can be pre-mixed proportionally prior to experiment as a stock solution) was first put onto the bottom of the centrifuge tube. Then, the middle layer, $250 \mu \mathrm{L}$ of a uniform mixture of $20 \%$ iodixanol (in final $25 \mathrm{mM} \mathrm{HEPES.KOH}, \mathrm{pH}$ 7.4, $400 \mathrm{mM} \mathrm{KCl}, 10 \mathrm{mM} \mathrm{MgCl}_{2}$ ), was laid gently without significant disturbing of the bottom layer. Lastly, the top layer, $50 \mu \mathrm{L}$ of buffer was laid on top. This tube of gradient mixture was then centrifuged at $50 \mathrm{~K}$ for $5 \mathrm{hrs}$ at $4^{\circ} \mathrm{C}$ on a SW55 rotor.

After centrifugation, 12 fractions, with about $57 \mu \mathrm{L}$ of each, were carefully collected from the top to the bottom. Fractions corresponding to the product were determined by nucleic acid electrophoresis using a gel containing $1.5 \%$ agarose and $0.5 \%$ SDS. Since the final complex has both Alexa 488 and Alexa 647 on it, product bands can be visualized by scanning the gel on a Typhoon Scanner (GE). In all of our preparation, the final, ring complex-SUV, floated up and equilibrated in fraction 2 or 3, right beneath the pink ring-free liposome band. Fractions containing final product were then combined and stored at $4^{\circ} \mathrm{C}$ for further use.

\section{Single molecule step-bleaching}

To confirm the actual number of full length VAMP2 templated in each ring complex-Anti tether i-liposome, we carried out single molecule step-bleaching experiment. 
Measurement was carried out in a flow-chamber set up. All cover glass (thickness No. 1.5, from Waldemar Knittel Glassbearbeitungs GmbH, Braunschwelg, Germany) was cleaned at Yale SEAS Cleanroom as previous described. ${ }^{3}$

During experiments, "ring complex-Anti tether i-liposome", obtained in section 8, was first diluted to the final concentration of $5-10 \mathrm{pM}$ liposome (equivalent to the ring-complex concentration, assuming one liposome bears exactly one ring) using buffer, $25 \mathrm{mM}$ HEPES.KOH, pH 7.4, $400 \mathrm{mM} \mathrm{KCl,} 2$ mM EDTA. This diluted liposome solution was then flown through the channel. Incubation time were adjusted so that, in each view, there were about $300-500$ spots that were deposited separately as single particle. After desired density of particles reached, $50 \mu \mathrm{L}$ of buffer (channel volume is about $2 \mu \mathrm{L}$ ), $25 \mathrm{mM}$ HEPES.KOH, $\mathrm{pH}$ 7.4, $400 \mathrm{mM} \mathrm{KCl}, 2 \mathrm{mM}$ EDTA, was flown through to rinse out any liposome that did not attach to the glass surface. TIRF video stream was then collected with an exposure time of $150 \mathrm{~ms}$. After acquisition, intensity traces for all single particles were extracted from the movie and the number of VAMP2s was estimated from the bleaching steps measured using ImageJ.

TIRF video streams were collected on a Nikon Ti Eclipse microscope using $642 \mathrm{~nm}$ excitation laser beam, Nikon 60x/1.45 oil objective, 1.5x model and EM-CCD camera (Andor iXon DU897E, $512 \times 512$ pixels). Filter set used to visualize full length VAMP2 cross-linked with Alexa647 is composed of zet640/20, zt640rdc, HQ660lp and ET 700/75m (from Chroma Technology Corp., Rockingham, VT).

\section{Transmission Electron Microscopy}

For negative staining transmission electron microscopy (TEM), a drop of sample ( $\sim 5 \mu \mathrm{L})$ was deposited on a glow discharged formvar/carbon coated copper grid (Electron Microscopy Sciences), incubated for 1-3 minute and blotted away. The grid was then washed briefly by $5 \mu \mathrm{L}$ of $2 \%(\mathrm{w} / \mathrm{v})$ uranyl formate solution and stained for 1 minute with another $5 \mu \mathrm{L}$ of $2 \%$ uranyl formate. Images were acquired on a JEOL JEM-1400 Plus microscope (acceleration voltage: 80 $\mathrm{keV}$ ) with a bottom-mount $4 \mathrm{k} \times 3 \mathrm{k}$ CCD camera (Advanced Microscopy Technologies). Electron micrographs were manually analyzed using ImageJ software.

11. SUV-SBL single event fusion assay on TIRF (measurements performed at Yale) Membrane fusion (lipid mixing) between templated-SUV (containing DNA-lipid tether) and tSBL (containing DNA-lipid tether) on a 4-channel ( $\times \mathrm{W} \times \mathrm{H}, 10 \mathrm{~mm} \times 2 \mathrm{~mm} \times 70 \mu \mathrm{m}$, each) 
flow-chamber setup described previously, ${ }^{3}$ except that the buffer conditions and the sample concentration were adjusted for our experiments as in the following section.

The 4-channel mask, SU-8 mold on silicon wafer and the PDMS blocks were all made at Yale SEAS Cleanroom. All the glass cover slips were cleaned at Yale SEAS Cleanroom, kept under dry vacuum in lab at RT, and were treated by plasma cleaning under air (on HARRICK, Plasma Cleaner/Sterilizer PDC-32G) for 4 mins right before use.

Briefly, during measurement, t-SUV (with DNA-lipid tethers), prepared as in section 7, were first diluted 10 fold using buffer, $25 \mathrm{mM}$ HEPES.KOH, pH 7.4, $400 \mathrm{mM} \mathrm{KCl}, 0.5 \mathrm{mM} \mathrm{MgCl}$, and then flown into each channel at a flow-rate of $0.8 \mu \mathrm{L} / \mathrm{min}$ for about $40 \mathrm{mins}$. t-SUV burst on the freshly treated glass cover slip (within 15 mins after plasma treatment) and formed supported lipid bilayers (SBL). Leftover liposome in solution was then rinsed out by flowing in buffer, 25 mM HEPES.KOH, pH 7.4, $400 \mathrm{mM} \mathrm{KCl,} 2 \mathrm{mM}$ EDTA, at $1.2 \mu \mathrm{L} / \mathrm{min}$ for about 45 mins. A few areas of SBL were then selected to check the fluidity of the membrane. Bilayers, that had no visible defects, no patches, and whose fluorescence (from NBD) could recover to at least $80 \%$ of the original intensity (under continuous $488 \mathrm{~nm}$ laser excitation) after photobleaching, were chosen to proceed for further measurement.

Templated SUV containing pre-organized v-SNARE and DNA-lipid tethers, prepared as in section 8, were then diluted to about $3 \mathrm{pM}$ (in terms of liposome) using buffer, $25 \mathrm{mM}$ HEPES.KOH, $\mathrm{pH}$ 7.4, $400 \mathrm{mM} \mathrm{KCl}, 2$ mM EDTA. Templated SUVs were then introduced by flow onto the SBL at a flow rate of $1.2 \mu \mathrm{L} / \mathrm{min}$. Membrane fusion was then recorded under continuous excitation of $532 \mathrm{~nm}$ laser (from lipid fluorophore, Rho-DOPE, signal).

\section{Data Analysis for Single Events}

Particles (liposomes in our case) for analysis were manually selected and the 2D trajectory of each liposome was tracked using Speckles TrackerJ plugin on ImageJ

(http://athena.physics.lehigh.edu/speckletrackerj/). Tethering, tightly-tethering and fusion stages of each event were defined manually. Occurrence of each stage was obtained directly from the number counting provided by Speckles plugin. This counting was then normalized by the area of study, the acquisition time and the concentration of liposome, which gave the normalized rate of each stage, as in equation 1. Percentage of stage $i$ that proceed to stage $j$ was defined as the ratio of the normalized rates of the two stages, as in equation 2 .

Normalized Rate $=\frac{\text { Number of events }}{\text { Area }\left(\mu m^{2}\right) \times \text { Acquisition Time }(s) \times \text { Liposome Concentration }(p M)}$ 
$\%$ of stage $i$ to stage $j=\frac{\text { Normalized Rate of stage } i}{\text { Normalized Rate of stage } j}$

Delay time between two stage $\mathrm{i}$ and $\mathrm{j}$ can be extracted from the tracking (obtained by Speckles plugin) trajectory between these two stages using Matlab codes published previously. ${ }^{3}$ Distribution of the delay time, presented as survival function, can also be obtained by running this Matlab program.

13. SUV-SBL single event fusion assay on TIRF (measurements performed at Harvard) For a better flow profile of vesicles and for an improved throughput of the assay, we adapted our microfluidic device to design an alternate version (Fig. S6). Flow devices were produced by pouring polydimethyl siloxane (PDMS) (Dow Corning) over a Prototherm 12120 mould manufactured using high-resolution stereolithography (Fineline prototyping). Dimensions of channels were $15 \mathrm{~mm} \times 500 \mu \mathrm{m} \times 80 \mu \mathrm{m}(\mathrm{LxWxH})$. The design change yielded an improvement in diffusion rate of lipids on the SBL layered in the channel, $2.83 \pm 0.61 \mu \mathrm{m}^{2} / \mathrm{s}(\mathrm{n}=37)$. All curve fitting and analysis for FRAP was performed using built in FRAP module for Zen 2011 software (Carl Zeiss AG). We had to reject $<15 \%$ channels due to visible "islands" of heterogeneity on the SBL.

Glass coverslip preparation and flow conditions for vesicles were similar to those described in 11. Data collected using both microfluidic device designs, Figs. 4 and S7-S9, are in excellent qualitative agreement with each other. Specifically, Fig. S7 highlights that the number of vesicles entering the LT stage is independent of the number of VAMP2 on templated SUVs. Similarly, the percentage of LT vesicles that enter the TT stage is also independent of the number of VAMP2 on these vesicles, Fig. S8. However, the percentage of vesicles in TT stage that undergo fusion (F stage) is dependent on the maximum number of externally oriented VAMP2 on templated SUVs. Our data shows this percentage to increase from $3.1 \%$ for no VAMP2 case to $12.39 \%$ for SUVs with $8 \times$ VAMP2, Fig. S9. Finally, delay time between the TT and F stages was plot as a survival function, Fig. S10.

\section{Calculations}

1. On SBL, there are about $2 \mathrm{t}$-SNAREs under each Ring-template The diameter of the origami-ring is $60 \mathrm{~nm}$, therefore, the area underneath is $A=\pi r^{2}=\pi \times$ $30^{2}=2826 \mathrm{~nm}^{2}$. Assuming that a phospholipid head group area is $0.65 \mathrm{~nm}^{2}$, we can calculate that under each ring-template, there are $\frac{2826}{0.65}=4.3 \times 10^{2}$ phospholipid molecules. Since the molar ratio of lipid and t-SNARE (mole/mole) we loaded was 2000:1, under each ring-template, there are about $\frac{4300}{2000} \approx 2 \mathrm{t}$-SNAREs under each ring-template. 
2. Estimated delay between tightly-tethered ("TT") stage and the docking stage ("D") As defined in the main text, the TT stage started when the tethered template-liposome immobilized on the SBL, and the D stage started when t- and v- SNAREs assembled with each other. The TT stage is characteristic as the immobilization of a vesicle. However, we do not have direct evidences for the $\mathrm{D}$ stage. Here, we want to use literature values to estimate the time delay between TT and D.

At the TT stage, template-vesicles were constrained close to the SBL and the separation distance between SUV and SBL was determined by the linker length of the DNA-lipid tethers (more details refer to a paper submitted separately). Within this small cylinder volume, there were $2 \mathrm{t}-$ SNARE molecules (calculated in above section). Then the assembly rate of the first v-SNARE binding to available t-SNARE can be calculated using known value of $k$ on.

In a proposed kinetic model of SNARE assembly pathway, ${ }^{4}$ after Syntaxin and SNAP25 slowly assembled into a $1: 1$ heterodemwe that provides a rapid binding site for synaptobrevin to form the ternary complex (Sytaxin-SNAP25/VAMP2). The $k_{\text {on }}$ of the assembly reaction of the ternary complex is measured to be $5 \times 10^{5} \mathrm{M}^{-1} \mathrm{~s}^{-1}$ (using soluble $\mathrm{t}$ - and $\mathrm{v}$-SNARE).

The DNA-lipid tethers we used in this study have 42 nucleotides (nt) on SUV and SBL side, respectively. Assuming the contour length of each nt is $0.5 \mathrm{~nm}$ (Smith, 1996), the maximum height of the cylinder covered under each ring-template ( $60 \mathrm{~nm}$ in diameter) is about $42 \mathrm{~nm}$. Then the volume of this cylinder is $V=h \pi r^{2}=42 \times \pi \times 30^{2}=119000 \mathrm{~nm}^{3}$.

Within this volume, the concentration of t-SNARE is

$C_{\mathrm{t}-\mathrm{SNARE}}=2 /\left[\left(6.02 \times 10^{23}\right) \times 119000 \mathrm{~nm}^{3}\right]=28 \mu \mathrm{M}$.

Then the concentration of 1 VAMP2 molecule on template-SUV is about $14 \mu \mathrm{M}$.

For the reaction:

$\mathrm{T}+\mathrm{V} \longrightarrow \mathrm{TV}$

$r=\frac{d[D]}{d t}=\mathrm{k}_{\mathrm{on}} \bullet[\mathrm{T}] \bullet[\mathrm{V}]-\mathrm{k}_{\mathrm{off}} \bullet[\mathrm{TV}] \approx \mathrm{k}_{\mathrm{on}} \bullet[\mathrm{T}] \bullet[\mathrm{V}]=175 \mu{\mathrm{M} . \mathrm{s}^{-1}}^{-1}$

Since under each vesicle, the cylinder volume was $119000 \times 10^{-24} \mathrm{~L}$, for each vesicle, the rate was $175 \times 10^{-6} \times 119000 \times 10^{-24}=2.1 \times 10^{-23} \mathrm{moles} / \mathrm{s}=13 \mathrm{molecules} / \mathrm{s}$.

Therefore, it should take about $100 \mathrm{~ms}$ for a tightly-tethered vesicle to proceed to the docking stage. 
In our experiment, the acquisition rate was $30 \mathrm{~ms}$ per frame. It is likely that after a few frames of immobilization, the docking stage started.

3. Some tether pairs start to engage at an average separation distance of about $25-30 \mathrm{~nm}$ The 5\% of PEG2000-DOPE in SBL and vesicles forms a 4-5 nm thick brush that forces the DNA to fully extend. Therefore, because each nucleotide contributes to a contour length of 0.5 $\mathrm{nm}$, the first 10 thymidines are forced to completely extend and the rest of the single stranded DNA (ssDNA) protrudes outside of the PEG brush. The remaining is a 53 nucleotide long freely jointed ssDNA chain. The end-to-end distance distribution is governed by the force/distance relationship, which is well described by a freely jointed chain model that incorporates stretchable Kuhn segments: ${ }^{5}$

$\operatorname{Ext}(\mathrm{F})=\mathrm{N}_{\mathrm{nt}} * \mathrm{a} *\left(\operatorname{coth}\left(\mathrm{Fb} / \mathrm{k}_{\mathrm{B}} \mathrm{T}\right)-\mathrm{k}_{\mathrm{B}} \mathrm{T} /(\mathrm{Fb})\right)(1+\mathrm{F} / \mathrm{S})$

Where $\operatorname{Ext}(\mathrm{F})$ is the end-to-end distance under a stretching force $\mathrm{F}, \mathrm{N}_{\mathrm{nt}}$ the number of nucleotides, a the contour length of a single nucleotide $(0.5 \mathrm{~nm}), \mathrm{b}$ the Kuhn segment length $(1.5$ $\mathrm{nm})$ and $\mathrm{S}$ the stretch modulus $(\mathrm{S}=800 \mathrm{pN})$.

Integrating this expression provides the energy $\mathrm{E}(\mathrm{Ext})$. The end-to-end distance probability density can then be directly expressed by:

$P(E x t)=\frac{E x t^{2} e^{E(E x t) / k_{B} T}}{\int_{0}^{N_{n} t^{a}} E x t^{2} e^{E(E x t) / k_{B} T} d E x t}$

From this probability density, one gets that $\sim 10 \%$ of the tethers extend $10 \mathrm{~nm}$ away from the PEG brush. Thus, some tether pairs will engage when the membranes are 25-30 nm apart (2 brush thicknesses +2 tether extensions).

4. The average separation distance after tether hybridization completes is about $15-20 \mathrm{~nm}$ After tether hybridization is complete, the first 10 thymidines are forced to extend within the PEG brush on each side and the remaining 64 thymidines are freely jointed ssDNA. With the same approach as in the previous section, the average distance is obtained by averaging the vertically projected end-to-end extension using the probability density (4). This average distance is $\sim 15-20 \mathrm{~nm}$.

5. Upon immobilization, templated SUVs are pulled towards SBL for about 10 15 $\mathrm{nm}$ We observed that immobilization of vesicles was usually accompanied by a step-increase of the fluorescence intensity (Fig. 4b).

Within the TIRF evanescent field, the fluorescence intensity of a spot exponentially decays with increasing distance of this spot relative to the focal plan, as quantified by equation (5): 


$$
I-I_{\text {background }}=\left(I_{0}-I_{\text {background }}\right) \cdot e^{-d / \delta}
$$

Where $I$ is the fluorescence intensity for a spot at a distance, $d$, relative to the focal plane; $\delta$ is the decay length (or penetration depth) of the TIRF evanescent field, which was measured to be 70 to $100 \mathrm{~nm}$, following a procedure previously described. ${ }^{6}$

Then for one spot at two distances, $d_{1}$ and $d_{2}$,

$$
\frac{I_{2}-I_{\text {background }}}{I_{1}-I_{\text {background }}}=e^{\left(d_{1}-d_{2}\right) / \delta}
$$

Therefore, the distance change, $\Delta d$, can be calculated by the fluorescence intensity change between these two distances,

$$
\Delta d=d_{1}-d_{2}=\delta \cdot \ln \left(\frac{I_{2}-I_{\text {background }}}{I_{1}-I_{\text {background }}}\right)
$$

We then picked 15 intensity time courses between the T and TT stages. Based on equation (6), the distance change upon immobilization is $10 \sim 15 \mathrm{~nm}$ with about $40 \%$ (SD) errors.

Table S1: Nomenclature and sequences of oligonucleotide

\begin{tabular}{|l|l|}
\hline & Sequence \\
\hline $\begin{array}{l}\text { Handle } i \\
\text { (5' end connects to the ring template) }\end{array}$ & $\begin{array}{l}\text { 5'-CCT TCT TCT TCT TAA CAA TCA AAA TTA TCT } \\
\text { ACC ACA ACT CAC-3' }\end{array}$ \\
\hline $\begin{array}{l}\text { Anti-handle } i \\
\text { (5' end crosslinked to the N-terminal of } \\
\text { VAMP2) }\end{array}$ & Thiol-5'-GTG AGT TGT GGT AGA TAA TTT-3' \\
\hline $\begin{array}{l}\text { Handle ii } \\
\text { (5' end connects to the ring template) }\end{array}$ & $\begin{array}{l}\text { 5'-CTA TCA CCT TCT AAC AAT TCA ACC TAC TAA } \\
\text { CAT AAT CAT CAC-3' }\end{array}$ \\
\hline $\begin{array}{l}\text { Anti-handle ii } \\
\text { (5' end crosslinked to Maleimide-DOPE) }\end{array}$ & $\begin{array}{l}\text { Thiol-5'-GTG ATG ATT ATG TTA GTA GGT TTT TTT } \\
\text { TTT TTT TTT TTT TTT TTT TTT TTT TTT TTT TTT T-3' }\end{array}$ \\
\hline $\begin{array}{l}\text { Tether i (t-Tether) } \\
\text { (5' end crosslinked to Maleimide-DOPE) }\end{array}$ & $\begin{array}{l}5^{\prime}-\text { Thiol-TTT TTT TTT TTT TTT TTT TTT TTT TTT TTT } \\
\text { TTT TTT TTT TTT TTC CTC TAC CAC CTA CAT CAC-3' }\end{array}$ \\
\hline $\begin{array}{l}\text { Anti-tether } \mathrm{i}(\mathrm{v}-\text {-Tether) } \\
\text { (3' end crosslinked to Maleimide-DOPE) }\end{array}$ & $\begin{array}{l}5^{\prime}-\text {-GTG ATG TAG GTG GTA GAG GAA TTT TTT TTT TTT } \\
\text { TTT TTT TTT TTT TTT TTT TTT TTT TTT TTT-thiol-3' }\end{array}$ \\
\hline
\end{tabular}


Table S2: Summary for analysis of fusion events. Data under analysis was acquired by using samples from two batches of preparation. For each batch of sample, three independent experiments were carried out. Membranes that passed quality control were selected for analysis.

\begin{tabular}{|c|c|c|c|c|c|c|}
\hline $\begin{array}{l}\text { Membrane } \\
\text { Area }\end{array}$ & $\begin{array}{l}\text { \# of } \\
\text { Vamp2 }\end{array}$ & $\begin{array}{l}\text { \# of } \\
\text { Fusion (F) } \\
\text { events in } \\
\text { about } 3 \\
\text { mins }\end{array}$ & $\begin{array}{l}\text { \# of Lose } \\
\text { Tethering } \\
\text { (LT) events } \\
\text { in about } \\
\text { 3mins }\end{array}$ & $\begin{array}{l}\text { \# of Tight } \\
\text { Tethering } \\
\text { (TT) events in } \\
\text { about } 3 \text { mins }\end{array}$ & TT/LT & F/TT \\
\hline 1 & 0 & 4 & 236 & 186 & 0.7881 & 0.0215 \\
\hline 2 & 0 & 4 & 168 & 146 & 0.869 & 0.0274 \\
\hline 3 & 0 & 11 & 278 & 266 & 0.9568 & 0.0414 \\
\hline 4 & 0 & 8 & 188 & 184 & 0.9787 & 0.0435 \\
\hline 1 & 1 & 1 & 174 & 115 & 0.6609 & $8.70 \mathrm{E}-03$ \\
\hline 2 & 1 & 2 & 192 & 130 & 0.6771 & 0.0154 \\
\hline 3 & 1 & 15 & 218 & 198 & 0.9083 & 0.0758 \\
\hline 4 & 1 & 29 & 184 & 172 & 0.9348 & 0.1686 \\
\hline 1 & 2 & 15 & 270 & 130 & 0.4815 & 0.1154 \\
\hline 2 & 2 & 28 & 228 & 178 & 0.7807 & 0.1573 \\
\hline 3 & 2 & 53 & 602 & 592 & 0.9834 & 0.0895 \\
\hline 4 & 2 & 22 & 302 & 294 & 0.9735 & 0.0748 \\
\hline 1 & 3 & 15 & 248 & 208 & 0.8387 & 0.0721 \\
\hline 2 & 3 & 30 & 204 & 192 & 0.9412 & 0.1563 \\
\hline 3 & 3 & 10 & 220 & 94 & 0.4273 & 0.1064 \\
\hline 4 & 3 & 14 & 166 & 114 & 0.6867 & 0.1228 \\
\hline 1 & 4 & 16 & 182 & 108 & 0.5934 & 0.1481 \\
\hline 2 & 4 & 12 & 138 & 90 & 0.6522 & 0.1333 \\
\hline 3 & 4 & 15 & 212 & 204 & 0.9623 & 0.0735 \\
\hline 4 & 4 & 22 & 216 & 208 & 0.963 & 0.1058 \\
\hline 5 & 4 & 56 & 388 & 374 & 0.9639 & 0.1497 \\
\hline 1 & 8 & 17 & 174 & 134 & 0.7874 & 0.1241 \\
\hline 2 & 8 & 14 & 172 & 146 & 0.8488 & 0.0959 \\
\hline 3 & 8 & 10 & 164 & 112 & 0.6829 & 0.0893 \\
\hline 4 & 8 & 10 & 160 & 110 & 0.6875 & 0.0909 \\
\hline 5 & 8 & 61 & 330 & 306 & 0.9273 & 0.1993 \\
\hline 1 & 16 & 43 & 388 & 384 & 0.9897 & 0.112 \\
\hline 2 & 16 & 77 & 538 & 534 & 0.9926 & 0.1442 \\
\hline 3 & 16 & 109 & 510 & 492 & 0.9647 & 0.2215 \\
\hline
\end{tabular}




\section{References:}

(1) Melia, T. J.; Weber, T.; McNew, J. A.; Fisher, L. E.; Johnston, R. J.; Parlati, F.; Mahal, L. K.; Sollner, T. H.; Rothman, J. E. J Cell Biol 2002, 158, 929.

(2) Shen, J.; Tareste, D. C.; Paumet, F.; Rothman, J. E.; Melia, T. J. Cell 2007, 128, 183.

(3) Karatekin, E.; Rothman, J. E. Nat Protoc 2012, 7, 903.

(4) Pobbati, A. V.; Stein, A.; Fasshauer, D. Science 2006, 313, 673.

(5) Smith, S. B.; Cui, Y.; Bustamante, C. Science 1996, 271, 795.

(6) Karatekin, E.; Tran, V. S.; Huet, S.; Fanget, I.; Cribier, S.; Henry, J. P. Biophys J 2008, 94, 2891. 
A. Liposomes containing SNAREs

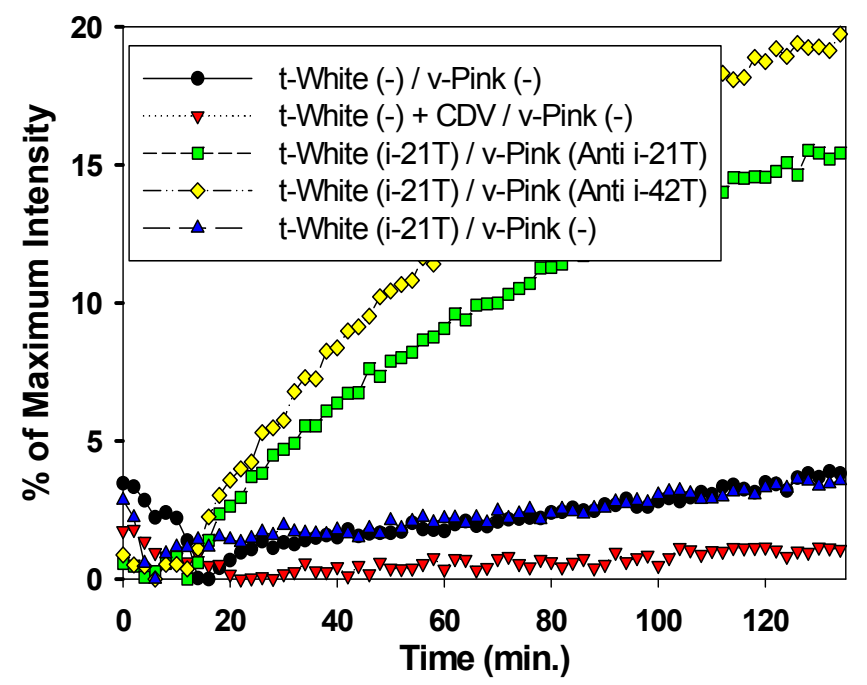

B.

Protein Free Liposomes

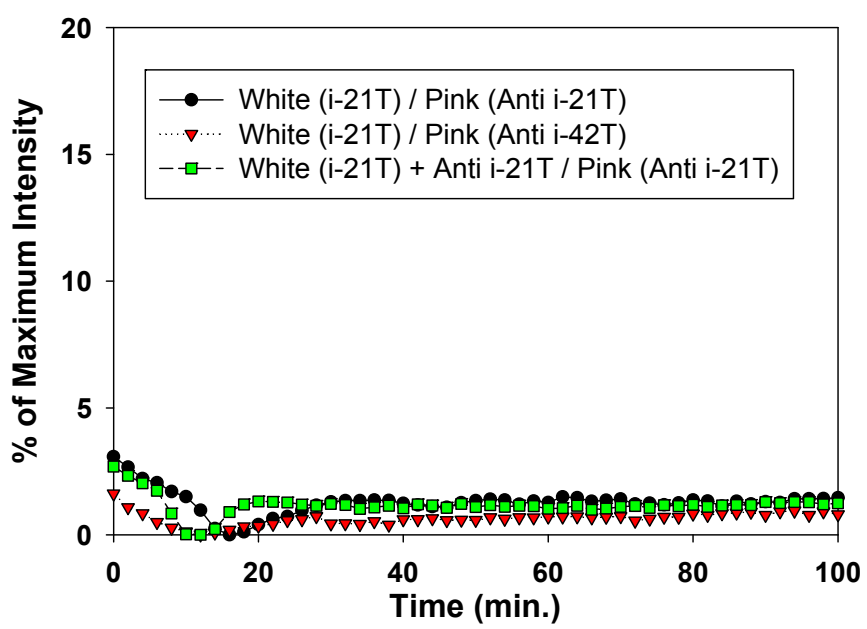

Fig. S1. Liposome-liposome fusion assay demonstrating that DNA-lipid tethers significantly increased SNARE-driven lipid mixing (A), but do not drive lipid mixing by themselves (B). For experiment details, please refer to our paper, "Accelerating SNARE-mediated membrane fusion by DNA-lipid tethers", Angewandte Chemie International Edition, 2015, DOI: 10.1002/anie.201506844. Briefly, a pair of DNA-lipid tethers were reconstituted into t- and v-liposome, respectively. Lipid-mixing caused by SNAREs was monitored by standard dequenching assay. 


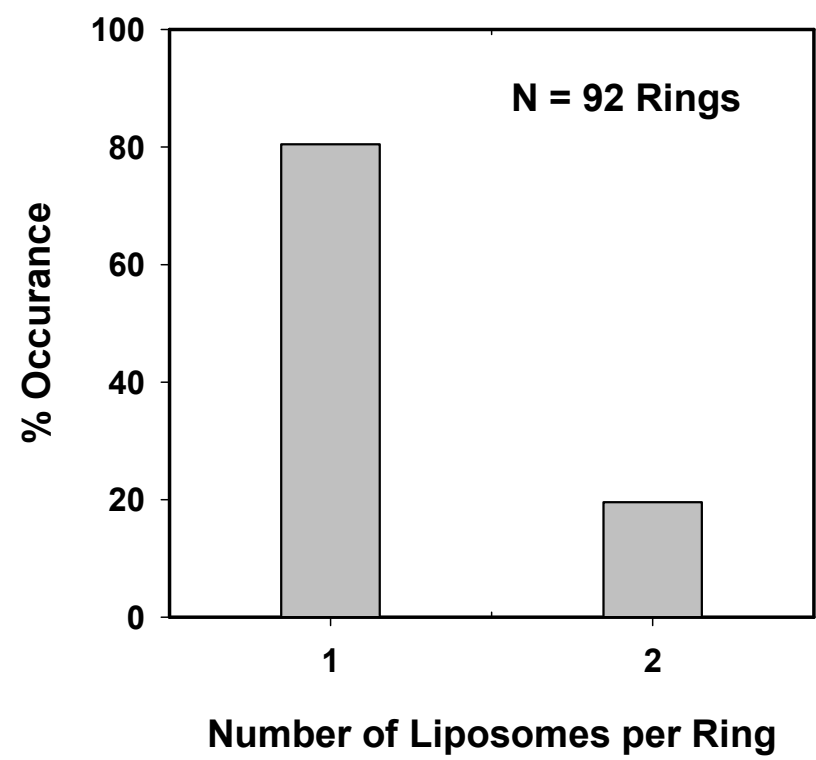

Fig. S2. Analysis of the TEM images of templated-SUVs. The number of liposome on each ring was counted manually. This histogram shows that about $80 \%$ of the rings carry exactly one vesicle. 
A.

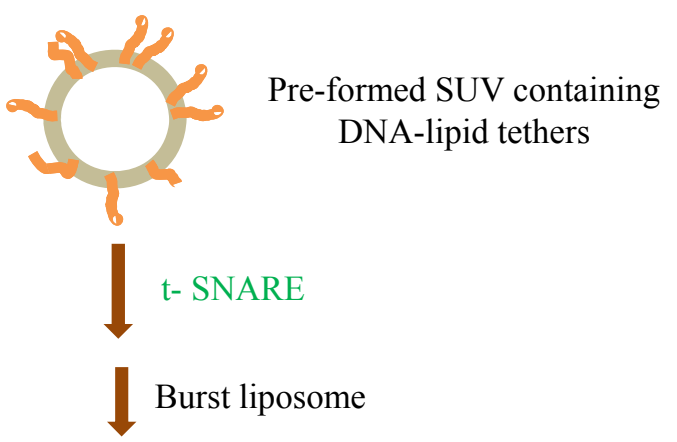

B.

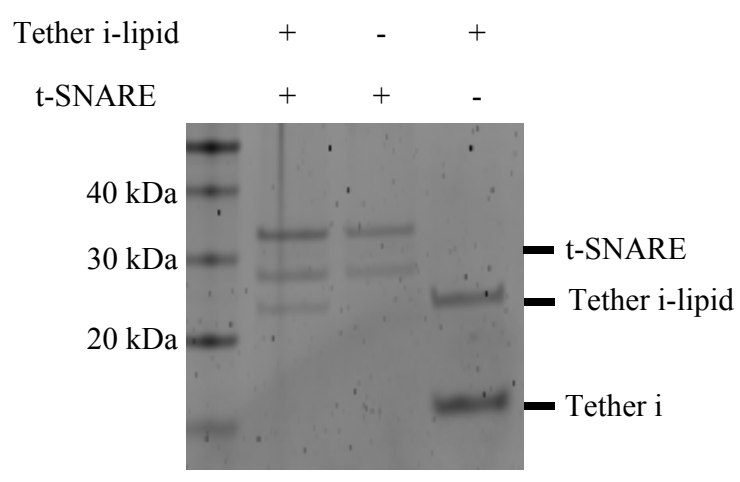

SBL containing full length t-SNARE and t-tethers

C.

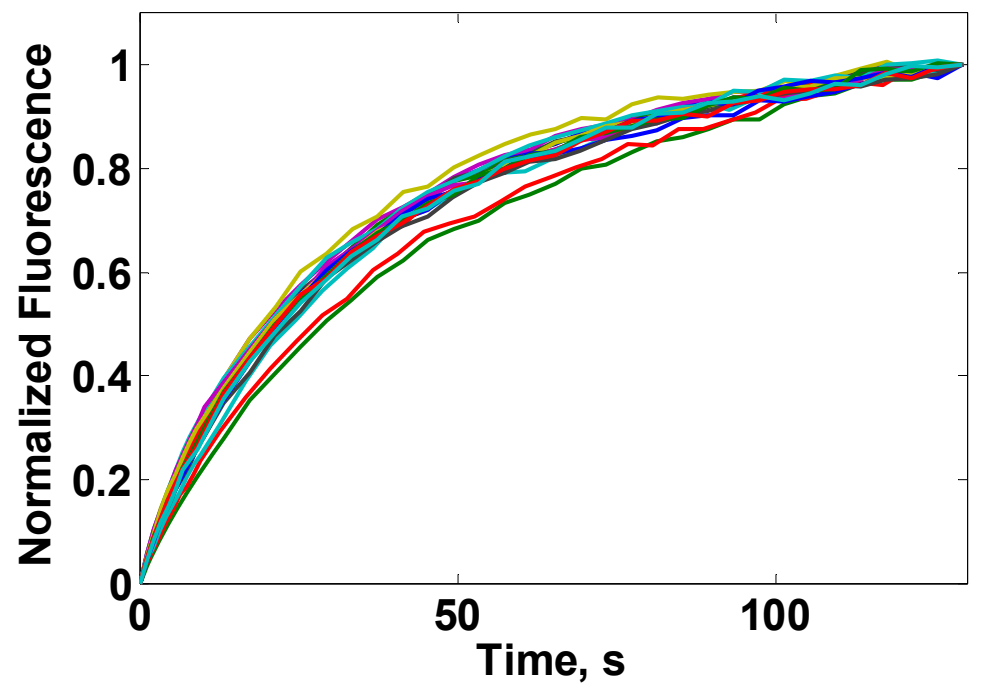

Fig. S3. Preparation of SBL that contains co-expressed Syntaxin1/SNAP25 (t-SNARE) and t-tethers. (A) SUVs containing t-tethers were pre-formed and purified, then tSNAREs were reconstituted into these SUVs following detergent-dialysis method. SBLs were prepared by bursting purified SUVs onto the surface of cleaned glass coverslips. (B) SDS-PAGE gel showing the presence of both t-SNAREs and t-tethers in SUVs. (C) Representative FRAP intensity-time courses of SBLs. All curves were fit to a function of the form $f=a\left\{\exp (-2 \tau / t) \times\left[I_{0}(2 \tau / t)+I_{1}(2 \tau / t)\right]\right\}$, where $I_{0}$ and $I_{1}$ are modified Bessel functions and $\tau=w^{2} /(4 D)$ is the characteristic diffusion time over a circle of radius $w$. The best fit parameters are $a=1.4$ and $\tau=30 \mathrm{~s}(R 2=0.99)$, implying a diffusion coefficient $D=2.4 \pm 0.4 \mu \mathrm{m}^{2} \mathrm{~s}^{-1}$ (Mean $\pm \mathrm{SD}$ ). Batch analysis was done using a Matlab code previously published [ E. Karatekin and J. E. Rothman, Nat. Protocol, 2012, 903] 
A.

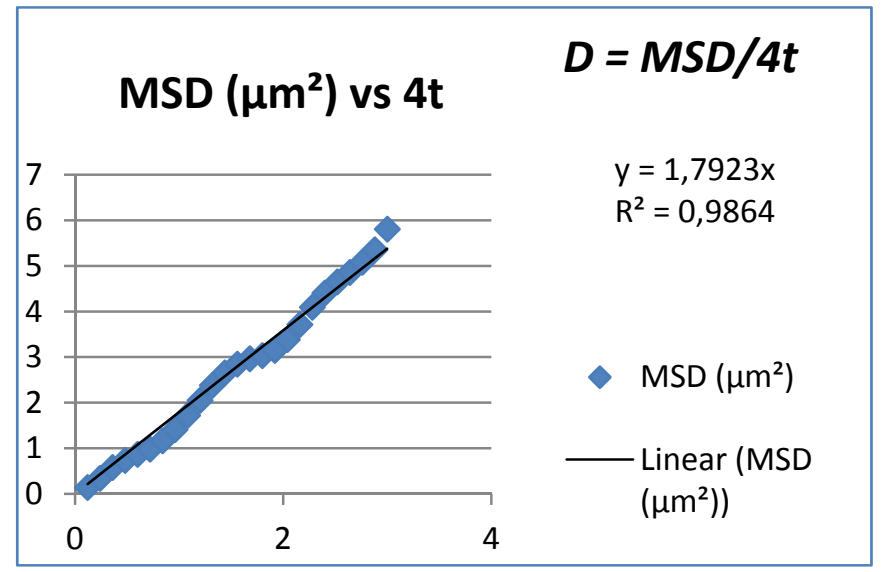

B.

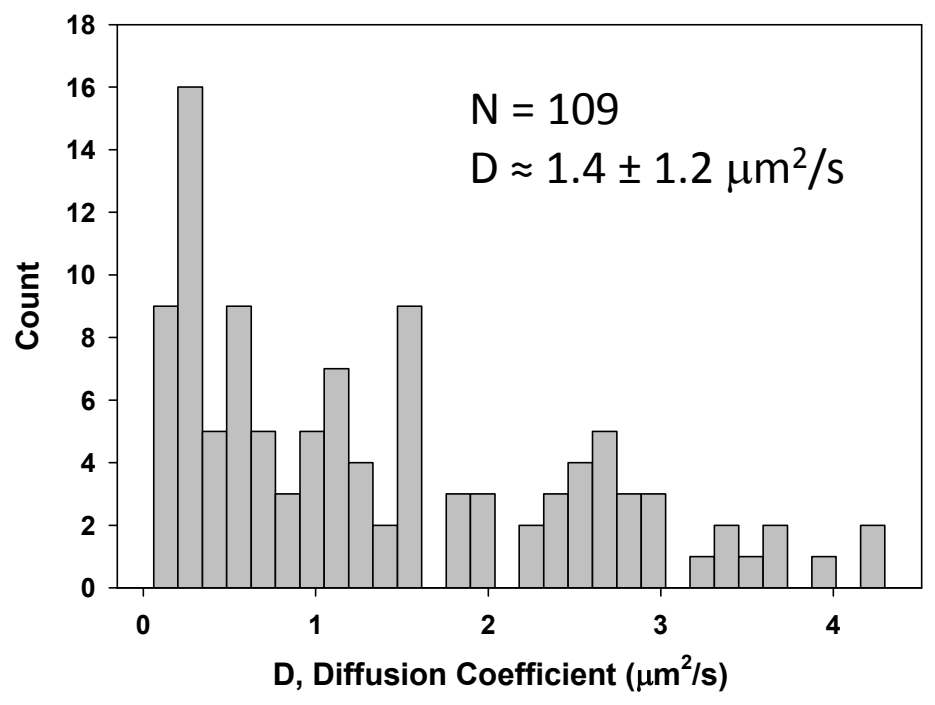

Fig. S4. Tethered vesicles are mobile on SBLs. (A) Representative example of mean square displacement (MSD) as a function of time. MSD was calculated based on the 2D diffusion trajectories obtained by Speckles plugin in ImageJ (see Suppl. Materials and Methods); (B) Histogram of diffusion coefficients of 110 randomly picked vesicles (with or without SNAREs). 


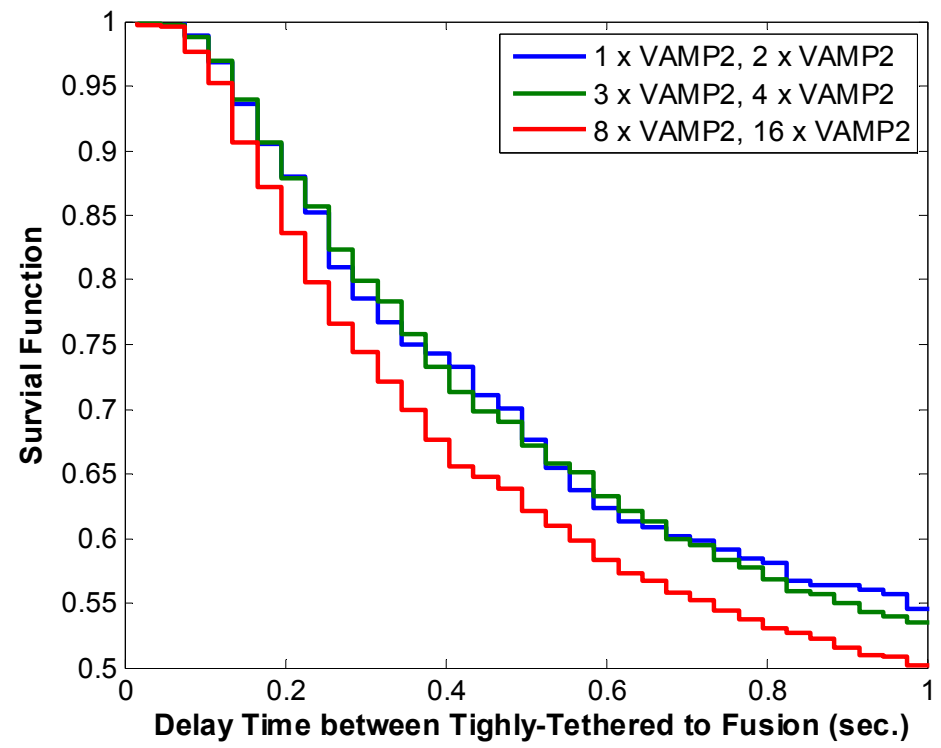

Fig. S5. Delay time between tightly-tethered stage and fusion. Distribution of delay time (represented as a survival function) between tightly tethering and fusion at different density of externally orientated VAMP2. 


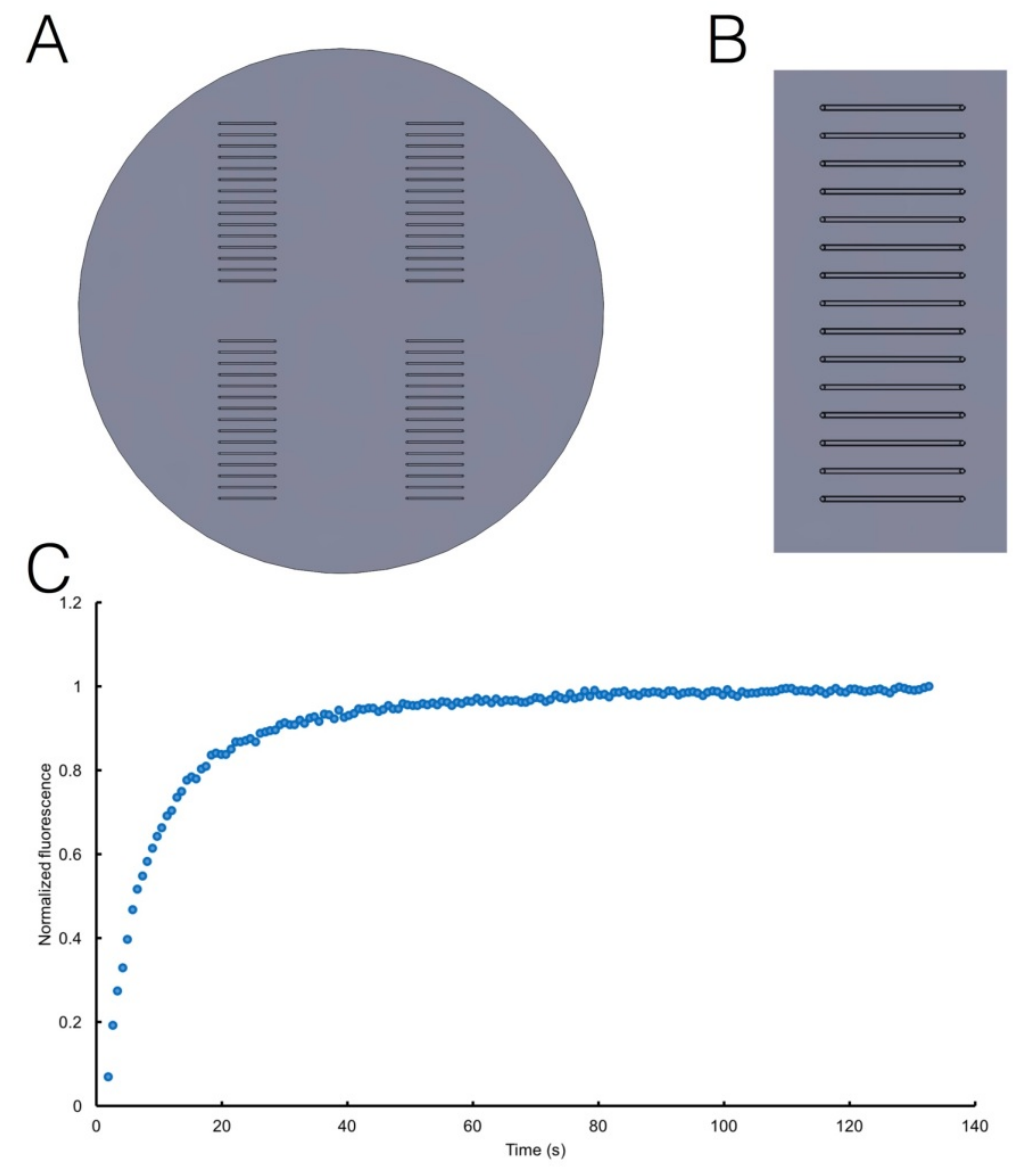

Fig S6. Template for the PDMS flow cell. (A) Design for a 15 channel flow cell. Dimensions of the plate are $140 \mathrm{~mm} \times 6.35 \mathrm{~mm}$. The plate yields 4 flow cells per run. The center to center distance between flow cells on the plate is $50 \mathrm{~mm}$. (B) close up view of one of the flow cells. Dimensions of each plate are $15 \mathrm{~mm} \times 500 \mu \mathrm{m} \times 80 \mu \mathrm{m}(\mathrm{L} \times \mathrm{W} \times \mathrm{H})$. For better flow profile and to reduce the strain at the inlet and outlet, each channel has a reservoir at both ends. (C) A representative FRAP measurement plot. 


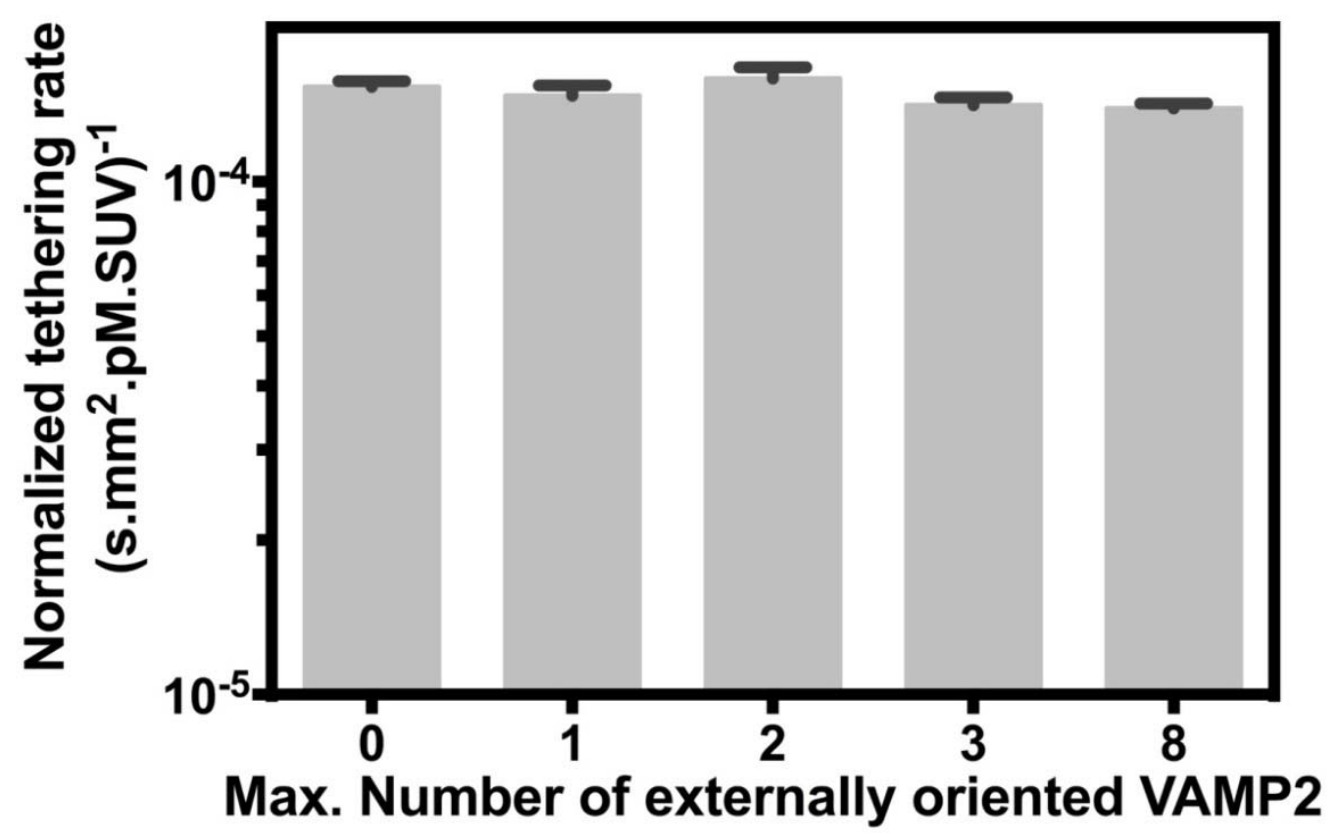

Figure S7. Number of tethering events normalized to the area of interest, concentration of the sample, and imaging time, expressed as a function of the maximum number of externally oriented VAMP2 on SUV. Data suggests tethering is independent of the number of VAMP2 


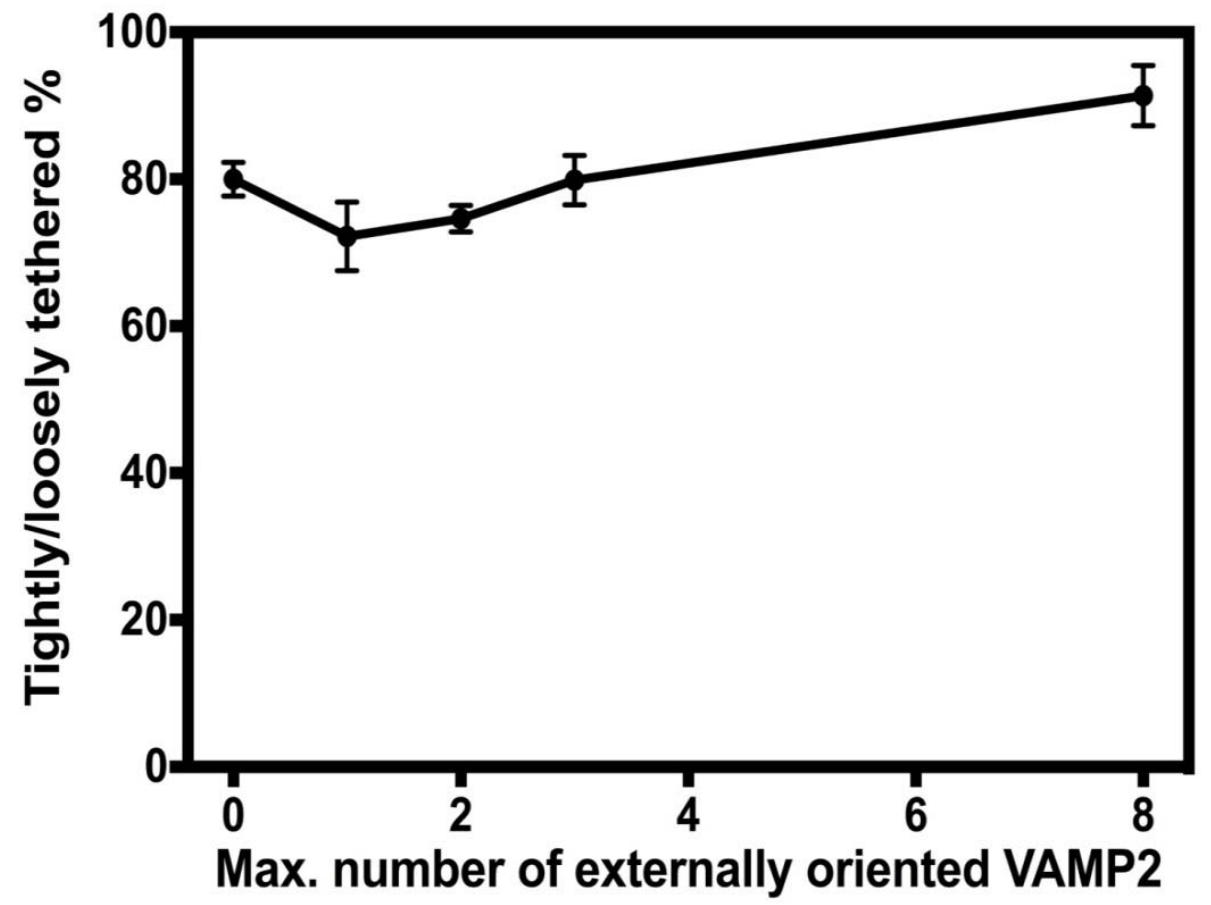

Figure S8. Percentage of loosely tethered SUVs that proceeded to tightly tethered state expressed as a function of maximum number of externally oriented VAMP2 on SUVs. A lack of correlation suggests that the tight tethering is not mediated by VAMP2 but rather by v- and t-tether pairs. 


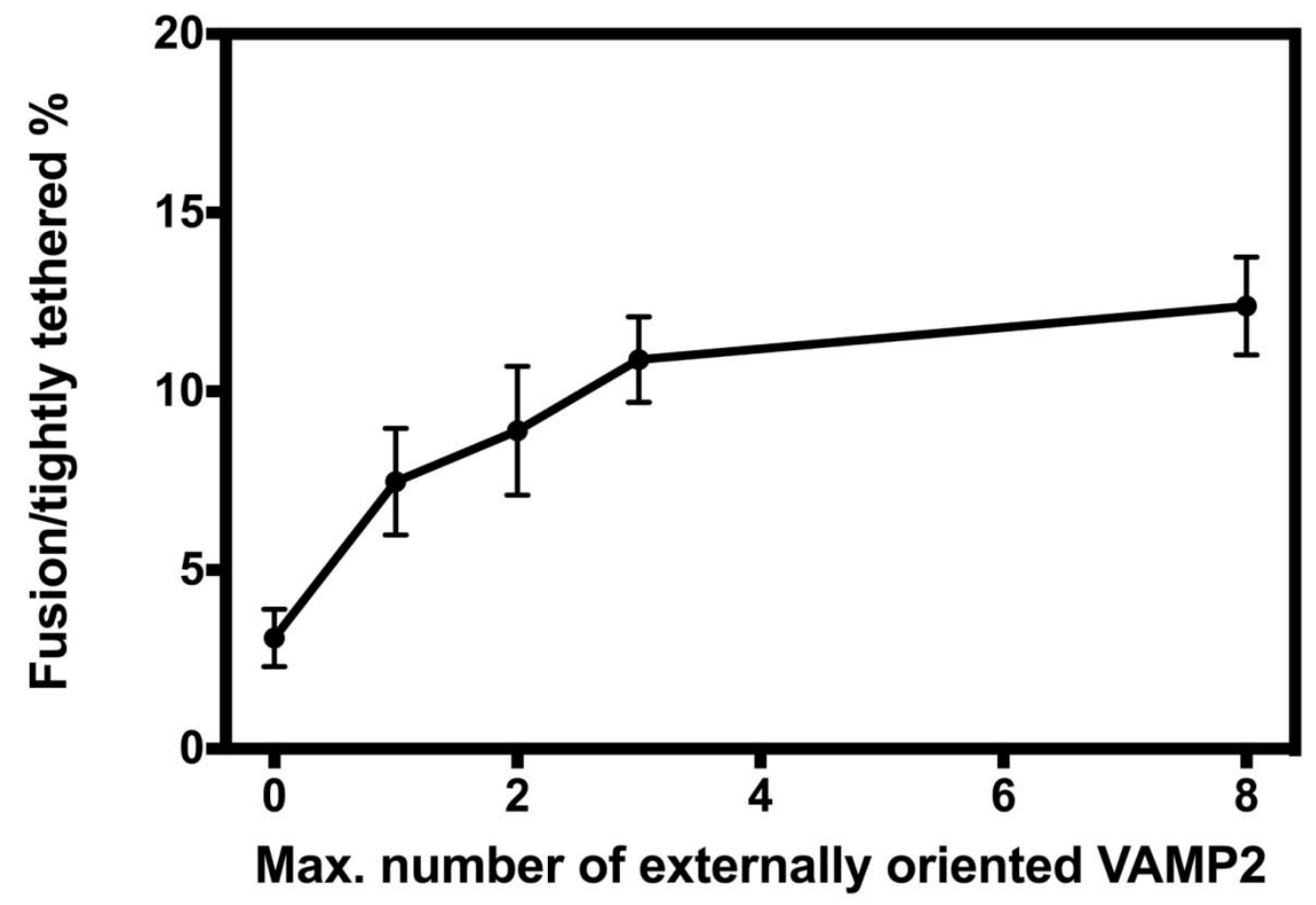

Figure S9. Percentage of tightly tethered SUVs that fused with the SBL expressed as a function of maximum number of externally oriented VAMP2 on SUVs. Data suggests one pair of SNAREs to be sufficient to drive SNARE-dependent fusion. 


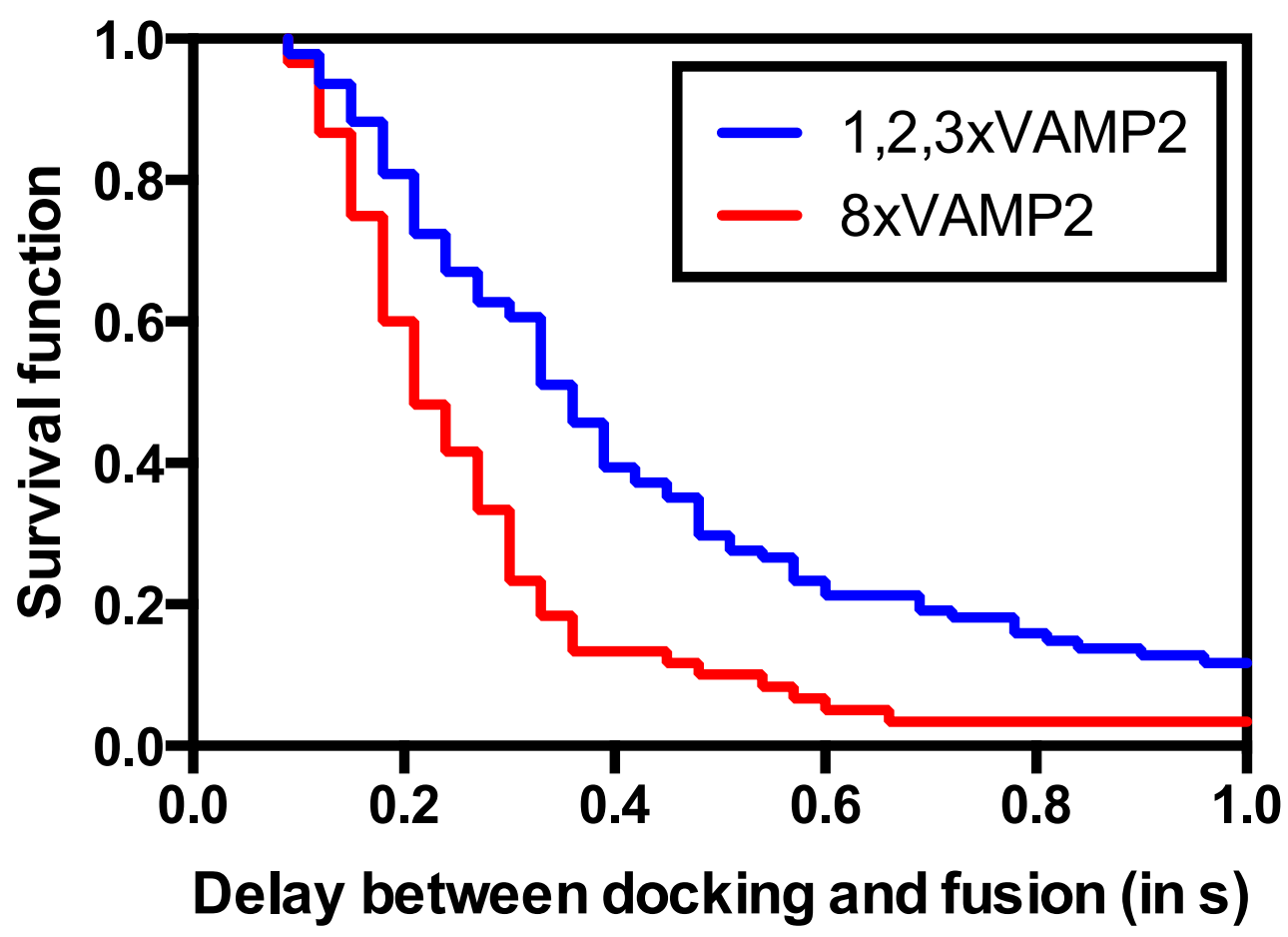

Fig. S10. Distribution of delay time between tightly-tethered and fusion stages expressed as a survival function. A majority of vesicles, $>80 \%$ for $1-3$ VAMP2 and $>93 \%$ for 8 VAMP2, fused within the first second of interaction with the SBL. 

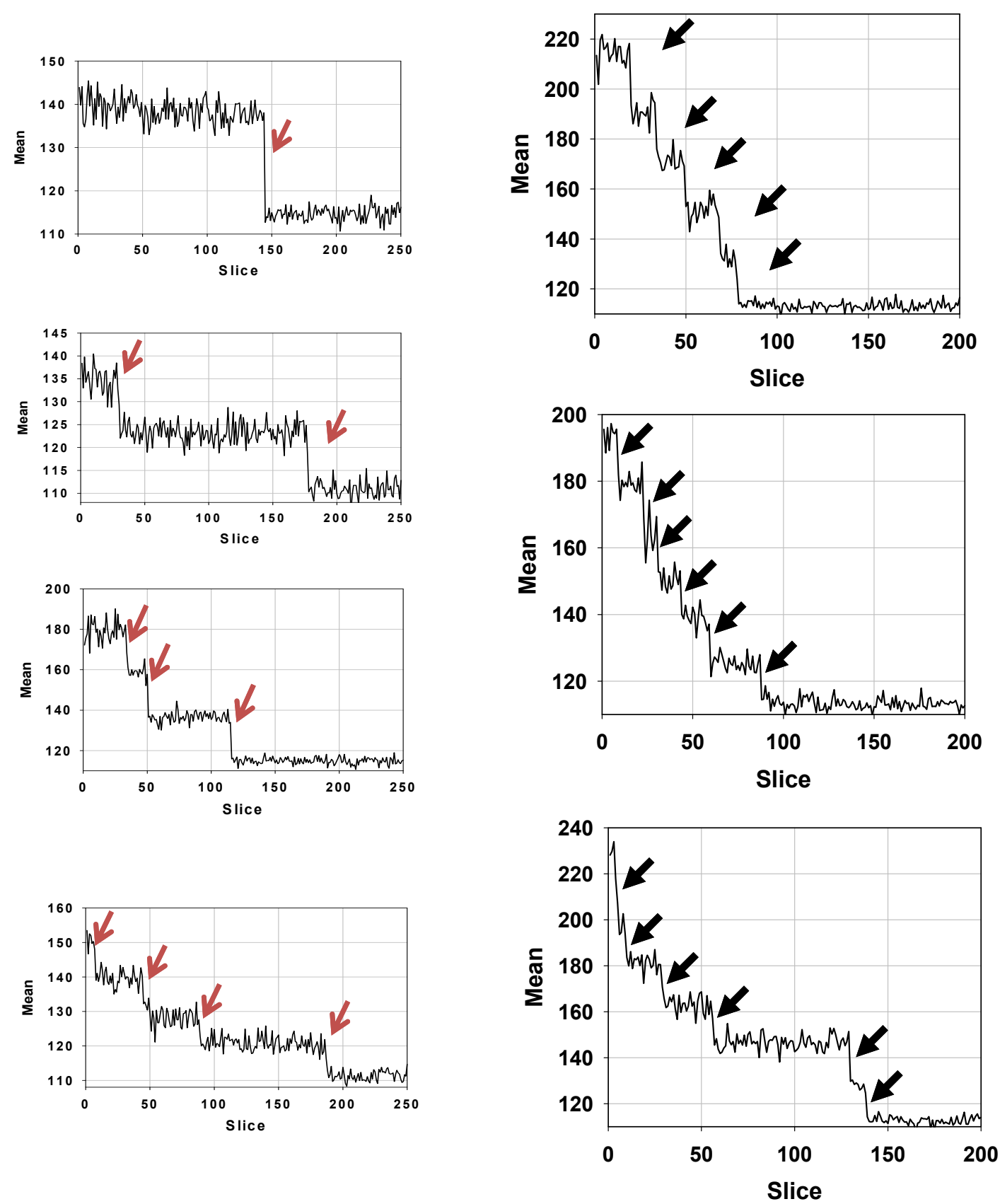

Figure S11. Examples of mean fluorescence intensity (area of $6 \times 6$ pixels) time courses showing that on our experimental setup we were able to resolve more than 4 bleaching steps. (data set are the same as in Figure 3). 

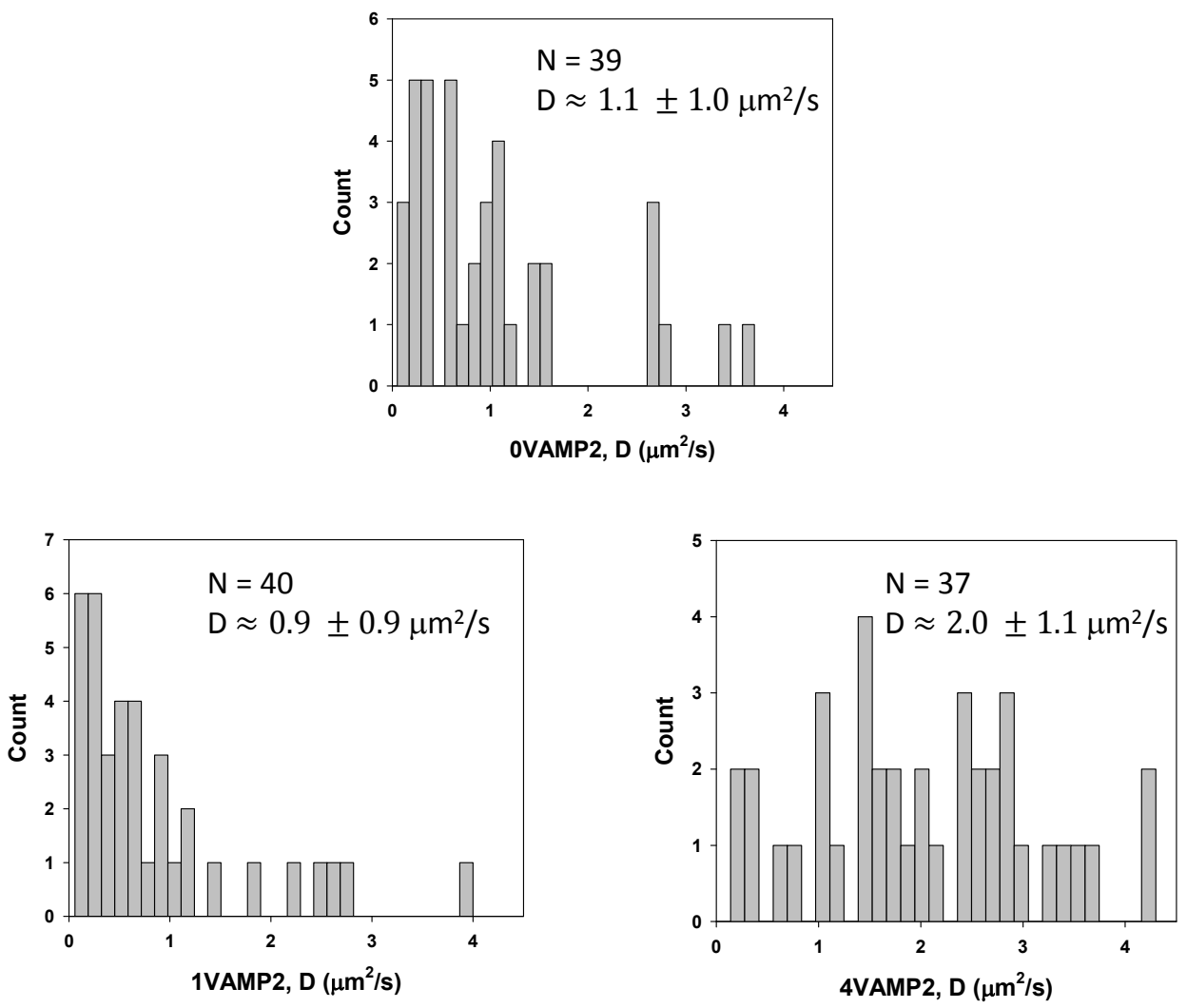

Figure S12. Histogram of diffusion coefficients of picked vesicles containing specified number of SNAREs. (data set are the same as in Figure S4b). 

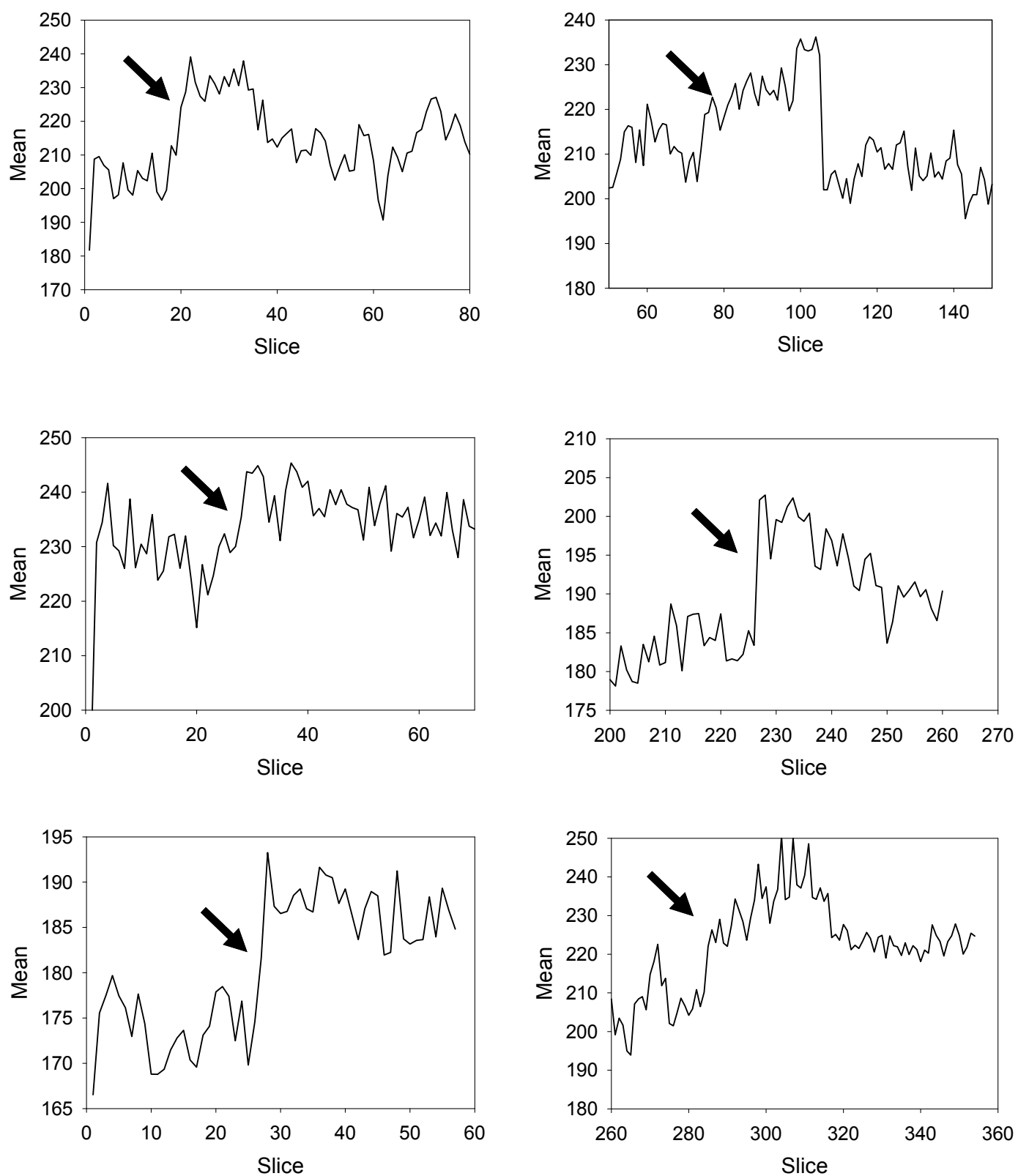

Figure S13. More examples of the mean fluorescence intensity time courses showing an intensity increase at the moment of immobilization of loosely tethered vesicles (entry into the TT stage). This increase indicates that when a vesicle were immobilized on the SBL, it was pulled toward the SBL. For detailed calculations, refer to Supplementary Information Calculation 5. (For all the traces, the background mean intensity is 130 .) 